\title{
Efectos de la regulación de la interacción diádica en tareas argumentativas
}

\author{
Nadia Soledad Peralta ${ }^{1}$, Néstor Roselli ${ }^{2}$ \\ Instituto Rosario de Investigaciones en Ciencias de la Educación (UNR)', \\ CONICET ${ }^{1}$, Universidad Nacional de Rosario ${ }^{1}$, Centro de Investigaciones en \\ Psicología de la Facultad de Psicología y Psicopedagogía (UCA)², Universidad \\ Católica Argentina ${ }^{2}$
}

\begin{abstract}
El trabajo tiene como objetivo analizar los efectos de la regulación interactiva de situaciones diádicas sobre las capacidades argumentativas de los sujetos en dos tipos de tareas: ordenamiento de causas de fenómenos sociales y toma de posición frente a situaciones dilemáticas. Se realizó un estudio cuasiexperimental, se comparó el pre-test y el pos-test de los sujetos luego de la interacción, constituyéndose dos grupos; díadas con asistencia de la interacción por parte del investigador (participación, evaluación y contra-argumentación) y díadas sin asistencia. Los resultados muestran que la asistencia produce efectos significativos en la competencia argumentativa individual y el tipo de tarea matiza el efecto seńalado, produciendo diferenciaciones. Se destaca la importancia de la regulación de la interacción sobre la argumentación.
\end{abstract}

Palabras clave: Interacción sociocognitiva, Argumentación, Contra-argumentación, Regulación de la interacción, Tipos de tarea.

Effects of the regulation of the dyadic interaction in argumentative tasks

The objective of this paper is to analyze the effects of the interactive regulation of dyadic situations on the argumentative abilities of the subjects in two types of tasks: ordering causes of social phenomena and taking position in front of dilemmatic situations. A quasi-experimental study was carried out, the pre-test and the post-test of the participants after the interaction were compared, constituting two groups; dyads with assistance from the interaction by the researcher (participation, evaluation and counter-argumentation) and dyads without assistance. The results show that the assistance produces significant effects in the

1 Doctora en Psicología por la Universidad Nacional de San Luis. Directora del programa Interdisciplinario de Análisis de Datos de la Universidad Nacional de Rosario. Investigadora Asistente del Consejo Nacional de Investigaciones Científicas y Técnicas (CONICET), Argentina. Instituto Rosario de Investigaciones en Ciencias de la Educación (IRICE-UNR). Dirección postal: 27 de febrero 210 bis (Ocampo y Esmeralda), Rosario (Santa Fe), Argentina (C.P. 2000). Contacto: nperalta@ irice-conicet.gov.ar

2 Doctor en Psicología. Investigador del Consejo Nacional de Investigaciones Científicas y Técnicas (CONICET), Argentina. Director del Centro de Investigaciones en Psicología de la Facultad de Psicología y Psicopedagogía de la Pontificia Universidad Católica Argentina (UCA), Buenos Aires, Argentina. Dirección postal: A. M. de Justo 1300, Buenos Aires, Argentina (C.P. 1107). Contacto: nestorroselli@uca.edu.ar 
individual argumentative competence and the type of task qualifies the indicated effect, producing differentiations. The importance of the regulation of interaction over argumentation is highlighted.

Keywords: Sociocognitive interaction, Argumentation, Counter-argumentation, Interaction regulation, Types of task

\section{Efeitos da regulaçáo da interaçáo em tarefas argumentativas}

O objetivo deste trabalho é analisar os efeitos da regulação interativa sobre as habilidades argumentativas dos sujeitos em dois tipos de tarefas: ordenar as causas dos fenômenos sociais e posicionar-se diante de situaçôes que contêm um dilema. Um estudo quase-experimental foi realizado, o pré-teste e o pós-teste dos sujeitos após a interação foram comparados, constituindo dois grupos; díades com assistência da interação pelo pesquisador (participação, avaliação e contra-argumentação) e díades sem assistência. Os resultados mostram que a assistência produz efeitos significativos na competência argumentativa individual e o tipo de tarefa qualifica o efeito indicado, produzindo diferenciaçôes. A importância da regulação da interação sobre a argumentação é destacada.

Palavras-chave: Interação sócio-cognitiva, Argumentação, Contra-argumentaçáo, Regulação da interação, Tipos de tarefas

Effets de la régulation de l'interaction dyadique dans les tâches argumentatives

L'objectif de ce travail est d'analyser les effets de la régulation interactive des situations dyadiques sur les capacités argumentatives des sujets dans deux types de tâches: ordonner les causes de phénomènes sociaux et se positionner face à des situations dilemmatiques. Une étude quasi expérimentale a été réalisée, le pré-test et le post-test des sujets après l'interaction ont été comparés, constituant deux groupes; dyades avec assistance de l'interaction du chercheur (participation, évaluation et contre-argumentation) et dyades sans assistance. Les résultats montrent que l'assistance produit des effets significatifs sur la compétence argumentative individuelle et que le type de tâche qualifie l'effet indiqué en produisant des différenciations. L'importance de la régulation de l'interaction sur l'argumentation est soulignée.

Mots-clés: Interaction socio-cognitive, Argumentation, Contre-argumentation, Régulation de l'interaction, Types de tâches 
Durante algunas décadas, diferentes investigaciones han estudiado los beneficios cognitivos de la interacción. En ese marco, la escuela de Ginebra enfatiza el análisis de la interacción sociocognitiva como escenario privilegiado para el estudio del progreso intelectual. Dicha corriente, dentro de la cual se enmarca el presente estudio, sostiene dos ideas básicas: la primacía estructural de la performance interindividual por sobre la individual y la experiencia de interacción como factor promotor del desarrollo cognitivo y del aprendizaje (Roselli, 1999).

En este contexto, Baker (1996 y 1998) sostiene que la argumentación supone un tipo de interacción en la cual los sujetos proponen a otro/s argumentos a favor o en contra de una determinada posición. En este sentido, son guiados para examinar y reflexionar sobre sus propios puntos de vista y los de sus compañeros respecto a una tarea en particular. El producto de esta interacción supone, en el mejor de los casos, un enriquecimiento de los puntos de vista individuales a partir de una comprensión más profunda del tema producto de la mediación de la opinión del compañero (Muller Mirza \& Perret-Clermont, 2009; Peralta \& Castellaro, 2018). Con todo, desde la perspectiva interaccionista, la argumentación es una actividad psicosocial que beneficia a los sujetos en términos cognitivos (Felton \& Kuhn, 2001), dado que los involucra en la posibilidad de un cambio epistémico y en la negociación de una fundamentación más elaborada (Leitao, 2000).

En esta perspectiva, el principio socioconstructivista sostiene que el marco ideal para la construcción del conocimiento es el diálogo argumentativo (Pérez-Echeverría, Postigo y García-Milá, 2016), por lo que se busca comprobar por un lado, la supremacía de las situaciones de interacción por sobre las individuales, y por otro lado, la importancia de la comunicación argumentativa por sobre la no argumentativa (Niño et al., 2019; Peralta, 2012; Peralta et al., 2012). 
Existe una diversidad de maneras de estudiar la argumentación, desde perspectivas más enfocadas en el análisis de lo formal, hasta aquéllas más centradas en el análisis del contenido. En este trabajo se comprende que la argumentación, como fenómeno social interactivo, puede ser regulada (asistida) o espontánea (no asistida).

Específicamente, los estudios que se han enfocado en evaluar la interacción y los procesos argumentativos han arrojado resultados diversos.

En investigaciones previas, Peralta y Roselli (2018) destacaron que los sujetos que han pasado por situación experimental de asistencia de la interacción por parte del investigador, han logrado mejores resultados en cuanto a la presencia de contra-argumentaciones en sus escritos individuales en comparación con sujetos que trabajaron en díadas sin asistencia en tareas dilemáticas. Asimismo, estos sujetos lograron tener mejores resultados en el nivel de participación, es decir, han aumentado el caudal de opiniones respecto a la situación planteada.

En otra investigación, Peralta y Roselli (2017) analizaron los distintos tipos de modalidades de argumentación en función del tamaño del grupo (díadas y tríadas) y del tipo de tarea (lectura de tablas de tres entradas, explicación causal de fenómenos físicos, toma de decisión respecto a situaciones morales dilemáticas y explicación causal de fenómenos sociales). Los resultados indicaron que en las díadas predominan las resoluciones argumentativas individualistas, es decir, un diálogo en el que el sujeto se limita a imponer su punto de vista, en tanto que en las tríadas prevalecen las resoluciones argumentativas dialógicas, es decir, un trabajo colaborativo con la intención de llegar a un consenso. En cuanto al tipo de tarea, las resoluciones argumentativas dialógicas predominaron en las tareas lógicas, en tanto que las resoluciones individualistas fueron más frecuentes en tareas de opinión. Más allá de estos resultados, según Bañales et al. (2014) uno de los retos fundamentales que se le presenta al sujeto es entender el tipo de tarea y de indagación disciplinar vinculada con la escritura argumentativa. Por lo general, las tareas argumentativas exigen a los estudiantes elaborar puntos de vistas sobre cuestiones controversiales, situadas en un contexto disciplinar 
específico, en este sentido es central ayudar a los sujetos a ubicarse contextual y disciplinarmente en la tarea que están realizando. Esto va en línea por lo planteado por Greco et al. (2017) quienes sostienen que el tipo de tarea (sobre todo en el ámbito educativo) es una variable central a la hora de estudiar la argumentación, dado que esta última está ligada al contexto en el que se desarrolla.

Finalmente, en un estudio cuasiexperimental, Peralta et al. (2012), evaluaron el efecto de la asistencia de la interacción en díadas y constataron que dicha asistencia tuvo efectos beneficiosos en los resultados de aprendizaje de los sujetos, así como en sus producciones escritas. Según Malpique \& Viega-Simao (2016), la argumentación es una habilidad de resolución de problemas que involucra operaciones cognitivas y retóricas como presentar y evaluar argumentos a favor y en contra de una afirmación y proporcionar datos que los apoyen. Estas habilidades propias de la argumentación (seleccionar, integrar y organizar los argumentos) es necesario aprenderlas y su aprendizaje potencia aprendizajes futuros.

Por otro lado, estas investigaciones que vinculan argumentación con respecto a la resolución de tareas pueden dividirse en dos clases, las que se orientan a estudiar la influencia de los objetivos de dicha tarea, sea persuadir o consensuar (Felton et al., 2015; García-Milá et al. 2013), y aquéllas centradas en estudiar el tipo de tarea, sean estas más abiertas o subjetivas, o más cerradas u objetivas (Peralta \& Roselli, 2017).

En lo que hace a este último aspecto, en las tareas cerradas $\mathrm{u}$ objetivas la argumentación está fundada en un criterio de correcto e incorrecto, en tanto que en las tareas abiertas o subjetivas la argumentación implica una toma de posición valorativa (Doise \& Mugny, 1991; Pohl \& Dejean, 2009).

Roselli (2004) encontró que las tareas de resolución de problema generaron mayor fluidez de intercambio cognitivo, mientras que las de explicación de fenómenos físicos requirió una mayor inversión de los participantes en la organización del intercambio específicamente cognitivo. 
En otro estudio, Roselli (2011) testeó diferentes tipos de tarea realizados por díadas, encontrando que las tareas abiertas llevan a construcciones auténticamente colectivas, mientras que las tareas cerradas o lógicas promueven en cambio una actividad de autorreflexión individual y negociación.

Aunque se reconoce que son importantes los diseńos cuasi experimentales para evaluar la influencia de distintos tipos de interacción sobre la argumentación, no son muchas las investigaciones que lo implementen. Precisamente a esto apunta el presente trabajo, cuyo objetivo es analizar los efectos de la regulación interactiva (asistencia) de situaciones diádicas argumentativas en comparación con situaciones diádicas espontáneas o no asistidas. Dichos efectos comparativos se evalúan en las producciones escritas individuales, donde se testeará la complejidad, la flexibilidad y la amplitud argumentativa después de la intervención, comparándose los dos tipos de condiciones: con y sin regulación en los dos tipos de tarea considerados (toma de posición frente a una situación dilemática "Toma de posición" y ordenamiento de posibles causas de fenómenos sociales "Ordenamiento").

\section{Método}

\section{Participantes}

La muestra estuvo constituida por 96 sujetos, todos estudiantes de primer año de diversas carreras de ciencias sociales. De este total, 48 trabajaron con la tarea "Toma de posición", divididos en 12 díadas con regulación del investigador (asistidas) 9, y 12 díadas que trabajaron libres (sin asistencia del investigador). Los 48 sujetos restantes realizaron la actividad "Ordenamiento" divididos en 12 díadas que trabajaron con regulación del investigador (asistidas) y 12 díadas que trabajaron libres. Todos los sujetos participaron voluntariamente en la investigación, firmaron el consentimiento informado y se les informó el uso anónimo de los datos. 


\title{
Materiales
}

Se diseñaron dos tareas. Una era denominada "Toma de posición”, en la cual los sujetos tenían que definir qué posición tomar ante cuatro situaciones dilemáticas, pudiendo optar por cuatro posibilidades: acordar o no con una determinada posición extrema o dos posiciones intermedias, una más próxima al acuerdo y otra más vinculada con el desacuerdo (ver Figura 1).

\begin{abstract}
Una pareja lleva tres aftos de casada y está esperando su primer hijo. Durante todo este tiempo Alicia y Nicolís han sido muy felices y se han dispensado una mutua sinceridad. En un cierto momento se produce una discusión entre ambos, la primera crisis de la pareja, que los deja a los dos resentidos. Es cuando entonces, en un viaje de negocios, y $\sin$ que lo haya buscado deliberadamente, Nieolas tiene una aventura sexual ocasional. Esto le genera mucha culpa y no sabe qué actitud asumir con su esposa, teniendo en cuenta el estado en que se encuentra. ¿Deberia contarle o no lo sucedido a su esposa?
\end{abstract}

\begin{tabular}{|l|l|}
\hline 1-Si, deberia contarle. & \\
\hline 2- Probablemente deberia contarle. & \\
\hline 3- Probablemente no deberia contarle. & \\
\hline 4- No deberia contarle. & \\
\hline
\end{tabular}

Figura 1. Ejemplo de la tarea de Toma de posición

La otra tarea, llamada "Ordenamiento", consistía en el ordenamiento que los sujetos debían hacer de siete posibles causas de fenómenos sociales, como por ejemplo la desocupación, siendo la categoría ordinal uno la causa más importante y siete la menos importante (ver Figura 2). 


A continuación te presentamos algunos fenómenos y sus posibles causas.
opciones de 1 a 7, correspondiendo 1 a la causa principal y 7 a la menos re
¿Cuál es la principal causa de la inseguridad, o sea de la gran cantidad de delitos
actualidad?
\begin{tabular}{|l|l|}
\hline La marginalidad y las deficientes condiciones de vida. & \\
\hline $\begin{array}{l}\text { La carencia de códigos minimos de convivencia por parte de los } \\
\text { delincuentes. }\end{array}$ & \\
\hline El desempleo y la falta de oportunidades laborales. & \\
\hline Leyes demasiado permisivas o de escaso cumplimiento. & \\
\hline La drogadicción. & \\
\hline Falta de control y acción policial. & \\
\hline La decadencia de la cultura del trabajo y la bonestidad. & \\
\hline
\end{tabular}

Figura 2. Ejemplo de la tarea de Ordenamiento

\section{Procedimiento}

Todos los sujetos de cada sub-muestra realizaron un pre-test que consistió en cuatro tareas de "Toma de posición" o cuatro de "Ordenamiento" según el caso similares a las expuestas en los ejemplos anteriores, requiriéndose solo respuestas sin argumentación, a los fines de detectar las orientaciones de base de los mismos.

Luego se conformaron las díadas a partir de las respuestas de los sujetos en ambos tipos de tarea ("Toma de posición" y de "Ordenamiento") apareando aquéllos que habían respondido de modo diferente, esto para garantizar la constitución de díadas heterogéneas.

Posteriormente, la situación experimental consistió en que los sujetos de las díadas discutieron sobre sus argumentos respecto de la tarea "Toma de posición" u "Ordenamiento", según dos modalidades: libre o espontánea (sin pautas de interacción), o regulada por el investigador. La consigna para ambos tipos de díadas fue la misma, "deben resolver esta actividad en conjunto y llegar a un consenso en la respuesta”, es decir, las díadas solo podían proponer una respuesta en común.

En el caso de las díadas reguladas, la intervención del investigador tuvo como objetivo apuntalar la interacción para mejorar la calidad argumentativa, interviniendo específicamente en tres aspectos: 
- la participación (estimular a dar una opinión y escuchar la del compañero)

- la evaluación (consultar a los sujetos si estaban o no de acuerdo [o no] con lo expuesto por el otro sujeto)

- contra-argumentación (requerir un argumento alternativo al ofrecido por el compañero)

Una vez finalizada la fase experimental todos los sujetos realizaron un pos-test individual, expresando por escrito las argumentaciones que en ese momento se les ocurrían plantear a cada uno para las tareas de "Toma de posición" u "Ordenamiento", según el caso, sin atenerse necesariamente a lo que se había discutido en conjunto oralmente en la fase anterior.

Las variables del estudio quedan sistematizadas en la tabla que se presenta a continuación.

\section{Tabla 1}

Variables del estudio

\begin{tabular}{c|c|c}
\hline $\begin{array}{c}\text { Variable } \\
\text { Independiente }\end{array}$ & Variable Interviniente & Variable dependiente \\
\hline $\begin{array}{c}\text { Tipo de regulación } \\
\text { diádica }\end{array}$ & Tipo de tarea & $\begin{array}{c}\text { Capacidad argumentativa individual } \\
\text { (post-test) }\end{array}$ \\
\hline Libre Asistida & $\begin{array}{l}\text { Toma de Ordenamiento } \\
\text { posición }\end{array}$ & Complejidad Flexibilidad Amplitud \\
\hline
\end{tabular}

Para medir el efecto del tipo de la regulación de la interacción, se comparan el pre-test individual escrito (solo de respuesta) con el post-test individual escrito (con argumentación) para ambas tareas. Se evaluará el pos-test individual escrito siguiendo el sistema de categorización que se presenta a continuación. 


\section{Análisis de datos}

Se analizaron tres dimensiones de la capacidad argumentativa: complejidad, flexibilidad y amplitud.

La complejidad argumentativa fue evaluada por dos indicadores (en ambos tipos de tareas):

a. cantidad de argumentos propuestos por el sujeto (el argumento es operacionalizado como una razón que justifique un punto de vista y se contabiliza uno por cada vez que aparezca), por ejemplo: "desde mi opinión, debería aceptar la propuesta, ya que las oportunidades muchas veces aparecen una sola vez en la vida, y predeterminan nuestro futuro, en este caso, profesional. Si está estudiando es porque los padres lo apoyan y quieren lo mejor para él y su futuro" o "decidi poner como causa más relevante de la desocupación las politicas gubernamentales errónea porque creo que las decisiones de un gobierno muchas veces favorecen a los sectores más ricos y dejan desprotegidos a los trabajadores.”.

b. formato de elaboración de dicho argumento, es decir, en primera persona ("Soy muy familiera y estar lejos de mis padres por cuatro años me resultaria muy dificil aunque me gustaría tener esa experiencia", "Elegí dicho orden porque si bien todos y cada uno son factores que repercuten a un alto nivel en los accidentes de tránsito, yo particularmente considero que las personas (el conductor) son los mayores responsables de ocasionar este tipo de situaciones por imprudencia y falta de conciencia") o tercera persona ("él ya habia cometido el error, entonces sería una acción de respeto contarle a su esposa lo sucedido y que luego ella lo tome como le parezca").

La flexibilidad también fue evaluada por dos indicadores:

a. tipo de respuesta dado por el sujeto (solo para la tarea "Toma de posición"): contundente ("si”" o "no") o relativizada ("probablemente sî" o "probablemente no")

b. cambio o no de respuesta del sujeto (elección de una respuesta igual o diferente en el post-test con respecto a la del pre-test). En la tarea "Toma de posición", dentro de las situaciones de cambio de respuesta entre el pre-test y el post-test existen tres posibilidades, tal como se muestra en la tabla a continuación. 


\section{Tabla 2}

Situaciones de cambio respecto al pre-test en la tarea "Toma de posición"

\begin{tabular}{cccl}
\hline Pre-test $\begin{array}{c}\text { Interacción (respuesta } \\
\text { conjunta elegida) }\end{array}$ & Post-test \\
\hline A & A & B & $\begin{array}{l}\text { Cambio de una posición } \\
\text { por otra no propuesta en la } \\
\text { interacción (Tipo 1). }\end{array}$ \\
A & B & C & $\begin{array}{l}\text { Cambio de una posición } \\
\text { por otra no propuesta en la } \\
\text { interacción (Tipo 2). } \\
\text { Cambio por la posición elegida } \\
\text { en la interacción (Tipo 3). }\end{array}$ \\
\hline
\end{tabular}

Dentro de las situaciones de no cambio de respuesta entre el pretest y el post-test existen dos posibilidades:

\section{Tabla 3}

Situaciones de no cambio respecto al pre-test en la tarea "Toma de posición"

\begin{tabular}{ccrl}
\hline Pre-test & $\begin{array}{c}\text { Interacción (respuesta } \\
\text { conjunta elegida) }\end{array}$ & Post-test & \\
\hline A & A & A & $\begin{array}{l}\text { El sujeto impone su respuesta } \\
\text { en la interacción y la mantiene } \\
\text { en el post-test. Es constante en } \\
\text { todo el proceso. }\end{array}$ \\
A & B & A $\begin{array}{l}\text { El sujetos mantiene su respuesta } \\
\text { en el post-test a pesar de la } \\
\text { interacción }\end{array}$ \\
\hline
\end{tabular}

En la tarea de "Ordenamiento" en la que los sujetos debían ordenar las siete causas disponibles del uno al siete en términos de importancia, el análisis se restringió a cualquier cambio que pudiera haber ocurrido en el post-test (respecto al pre-test) en la causa ubicada en el primer (uno) y último lugar (siete). 
Finalmente, la amplitud argumentativa, comprendida como la medida de la extensión del discurso argumentativo, se evaluó por la cantidad de palabras en las respuestas argumentativas emitidas por cada sujeto en ambos tipos de tareas.

\section{Resultados}

\section{Complejidad argumentativa}

Con respecto a la cantidad de argumentos en la tarea "Toma de posición", se pudo observar que los participantes que pasaron por interacción en díadas con asistencia emitieron un total de 34 argumentos en sus escritos (pos-test), con una media de $1.42(D E=.48)$ por persona. Si bien este total es superior a los argumentos emitidos por los sujetos que no experimentaron asistencia en la interacción (27, $M=1.13, D E=0.85$ ), no existen diferencias estadísticamente significativas para estos valores. Así, se puede afirmar que la asistencia no tuvo un efecto significativo en la cantidad de argumentos emitidos por los participantes.

En el caso de "Ordenamiento", la cantidad de argumentos propuestos por los sujetos que pasaron por interacción en díadas con asistencia $(68, M=2.83, D E=.76)$ fue superior a la cantidad de argumentos elaborados por los sujetos que no pasaron por asistencia en la interacción (56, $M=2.33, D E=0.56)$. En este caso la diferencia entre ambas condiciones según la prueba de $\mathrm{T}$ Student es significativa $(t=2.584 ; \mathrm{gl}=46 ; p<.05)$.

Con relación al formato en el que se expresó el argumento (en primera o tercera persona) se encontró, para la tarea "Toma de posición", que los sujetos de las díadas sin asistencia expresaron sus argumentos mayormente en tercera persona $(70.8 \%)$, es decir, siguieron haciendo referencia al personaje de la tarea(por ejemplo "Luis debería irse de viaje porque...”), mientras que la mayor parte de los sujetos de las díadas con asistencia (58.3\%) escribieron sus argumentos en primera persona (por ejemplo, "Yo me iría de viaje porque...."). Las diferencias entre los 
valores de ambos tipos de condiciones son estadísticamente significativas $\left(X^{2}=4.148 ; \mathrm{gl}=1 ; p<.05\right)$. Este resultado pone de relieve un claro efecto de la regulación interactiva de la situación diádica (asistencia) en la que los sujetos fueron específicamente instruidos para escuchar la posición del compañero, evaluarla y expresar su propio punto de vista. Por lo tanto, durante la escritura individual posterior no hacen más que seguir actuando de esa manera, involucrándose personalmente en la situación y defendiendo claramente una posición personalizada ("yo haría", "yo creo") ante un otro "virtual".

En cambio, en la tarea "Ordenamiento", todas las respuestas fueron elaboradas en primera persona "yo elegí esta orden porque...", "decidí poner como causa”. Probablemente esto fue así porque la tarea planteaba directamente una toma de posición propia, ya que el sujeto debía ordenar (y por lo tanto manifestar) las causas del fenómeno social según su criterio personal (incluida su justificación).

\section{Flexibilidad}

Otro efecto notable de la asistencia en la tarea "Toma de posición" fue el tipo de respuesta ofrecida por el sujeto. Mientras que los sujetos que pasaron por díadas sin asistencia emitieron en su mayoría una respuesta contundente ("Sî" o "No"), los que recibieron asistencia emitieron mayormente una respuesta relativizada ("Probablemente si" o "Probablemente no").

La diferencia entre cantidad de respuestas relativizadas $(66.7 \%$ en las díadas asistidas y $25 \%$ en las díadas sin asistencia) y cantidad de respuestas contundentes $(33.3 \%$ en las díadas asistidas y $75 \%$ en las díadas sin asistencia) es significativa según la prueba de Chi cuadrado $\left(X^{2}=8.392 ; g l=1 ; p<.01\right)$.

Parecería ser que la intervención en torno a la escucha, evaluación y posicionamiento recíprocos llevó a los sujetos a relativizar sus posiciones. Esto significaría que, al producir una respuesta, los sujetos tuvieron en cuenta la posible existencia de puntos de vista alternativos al suyo. 
Con respecto a los cambios registrados entre pre-test y post-test, como puede observarse en la figura 3, los sujetos que pasaron por interacción regulada tendieron a realizar cambios de tipo $\mathrm{ABB}(10,41.7 \%)$, es decir, cambiar su posición inicial por la trabajada y consensuada con el compañero durante la interacción, 3 cambios de tipo ABC (12.5\%), 5 no-cambio de tipo AAA (20.8\%) y 6 no-cambio ABA (25\%), mientras que los sujetos de las díadas sin regulación presentaron una mayor cantidad de no-cambio de tipo AAA $(11,45.8 \%)$, es decir, que mantuvieron constantes su posición durante todo el proceso, 4 no-cambio ABA (16.7\%), 6 cambios ABB (25\%) y 3 cambios ABC (12.5\%).

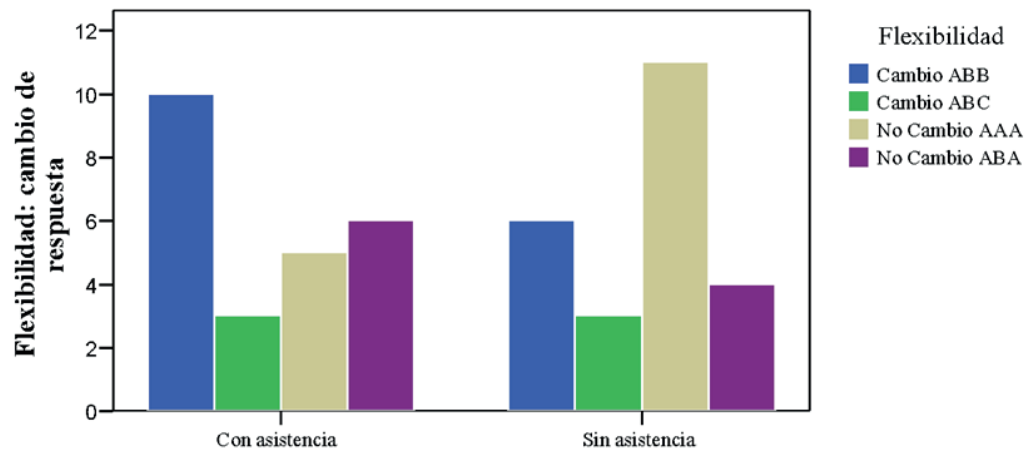

Tipo de Díada

Figura 3. Cambio de respuesta entre pre-test y post-test en función del tipo de condición en la que participó el sujeto (con asistencia y sin asistencia)

En la tarea "Ordenamiento" los resultados indicaron que todos los sujetos de ambos grupos de díadas (asistidas y no asistidas) cambiaron sus respuestas luego de la interacción con el compañero. Esto podría llevar a pensar que toda situación de interacción (sea asistida o no asistida) produce un efecto de cambio, aunque quizás no se deba tanto a la interacción propiamente dicha, sino a la naturaleza compleja de la tarea, la cual implicaba establecer un ordenamiento entre siete posibles causas. 


\section{Amplitud argumentativa}

En la tarea "Toma de posición", la cantidad de palabras emitidas por los sujetos que realizaron la tarea sin asistencia de la interacción fue de 1089, con $M=45.38$ ( $D E=32.15$ ), mientras que los sujetos que sí tuvieron asistencia de la interacción emitieron en total 1053 palabras, con $M=43.88$ ( $D E=19.38$ ) por sujeto. Como se observa no hay diferencias significativas respecto la cantidad de palabras emitidas en ambas condiciones, aunque hay que remarcar la notoria diferencia de los desvíos, lo que indicaría un efecto de homogeneización en cuanto a la cantidad de palabras en los sujetos de la condición asistida ( $t=-.196$; $g l=46 ; p>.05)$.

Esto mismo se constata en la tarea "Ordenamiento", donde la cantidad de palabras emitidas por los sujetos que realizaron la tarea sin asistencia de la interacción fue de 2133, $M=88.88$ ( $D E=23.83)$, mientras que los sujetos que sí tuvieron asistencia de la interacción emitieron en total 2335 palabras con un promedio de $M=97.29$ ( $D E=11.78$ ) por sujeto. Aquí también la diferencia entre los desvíos habla de un efecto homogeneizador en la amplitud argumentativa individual (post-test) de la condición asistida $(t=1.551 ; g l=46 ; p>.050)$.

Con todo, lo que más interesa del análisis de la amplitud argumentativa es verificar si el patrón de homogeneización promovido por la asistencia en lo que hace a la distribución equitativa de la cantidad argumentativa entre los sujetos de cada díada, se mantiene en la performance individual del post-test, ya que puede hipotetizarse que este patrón de homogeneización inducido por la intervención experimental se traslada posteriormente al comportamiento argumentativo individual.

Para analizar esto basta con medir la diferencia entre la cantidad de palabras emitidas en el post-test por ambos sujetos de cada díada (diferencia intra-díada). Si la homogeneización experimental se traslada al plano de la performance individual, el promedio de las diferencias intra-díadas de la condición asistida debería ser menor que el de los sujetos no asistidos. Esto fue lo que se encontró, ya que el promedio de 
estas diferencias fue de $12.45(D E=6.43)$ para los sujetos de las díadas de la condición asistida y de $21.22(D E=19.09)$ para los de la condición no-asistida en la tarea de "Toma de Posición", y de $14.92(D E=10.57)$ y de $20.75(D E=15.01)$ respectivamente en la tarea "Ordenamiento".

\section{Discusión}

El objetivo de este trabajo fue evaluar el efecto de la regulación de la interacción (asistencia del investigador) sobre la capacidad argumentativa individual de los sujetos. Dicha capacidad fue evaluada siguiendo tres indicadores: complejidad, flexibilidad y amplitud argumentativa.

Con respecto a la complejidad se pudo observar que en la tarea "Toma de posición" la regulación del investigador no tuvo un efecto significativo sobre la cantidad de argumentos emitidos; sin embargo, en la tarea "Ordenamiento" la intervención sí tuvo significatividad, dado que los sujetos de las díadas con regulación presentaron mayores cantidades de argumentos que los sujetos de las díadas sin regulación. Eso es coincidente con los resultados obtenidos por Peralta y Roselli (2018) quienes sostienen que la regulación estimula mayor cantidad de participación y expresión de puntos de vista.

La cantidad de argumentos en "Ordenamiento" fue mayor. Esto probablemente haya sucedido por la estructura de la tarea, en la que los sujetos estaban de alguna manera obligados a argumentar uno por uno el orden jerárquico elegido para las siete causas propuestas del fenómeno social en cuestión, mientras que en "Toma de posición", al tener que elegir una opción entre cuatro posibilidades, con un mínimo de un solo argumento, era suficiente. Esta mayor complejidad de "Ordenamiento" contribuyó a que, al estimular la regulación el intercambio diádico, hubiera mayor cantidad de argumentos explicitados.

En cuanto al formato en el que se expresaron los argumentos, se pudo observar en general que los sujetos expresaron mayormente sus puntos de vista en primera persona, apropiándose en forma personalizada de una posición. Esto ocurrió sobre todo en el caso de las díadas 
con regulación en "Toma de posición", donde los sujetos que tuvieron asistencia fueron los que elaboraron mayormente las argumentaciones de sus escritos en primera persona. Esto puede ser considerado efecto de la regulación, dado que en la misma los sujetos fueron instruidos para escuchar la opinión del compañero, evaluarla y expresar su propio punto de vista. Esto explica por qué en la escritura individual de posttest de los sujetos de esta condición, las respuestas se iniciaban con la afirmación típica "yo haría...", en lugar de referirse a lo que haría el personaje protagonista del dilema presentado.

En cambio, en "Ordenamiento", en dichas producciones individuales de post-test los sujetos expresaban en general sus opiniones en primera persona, tanto los de la condición con regulación como los de la no regulada. Probablemente esto se vincule más con la tarea que con la regulación, dado que mientras que en "Toma de posición" la tarea hacía referencia específica a una tercera persona (el personaje del dilema), en "Ordenamiento" la tarea no hace referencia a un personaje, directamente apela al sujeto que está realizando la tarea, pidiéndole directamente su opinión en relación al fenómeno que se está evaluando.

Estos resultados van en la misma línea que los obtenidos por Peralta y Roselli (2017) quienes sostienen que las tareas de opinión en general propician argumentaciones dialógicas más involucrantes desde el punto de vista personal, aunque habría que agregar, de acuerdo a las conclusiones del presente estudio, que además del tipo de tarea la regulación de la interacción en términos de estímulo de la escucha, de la evaluación y de la contra-argumentación,producen también un efecto personalizante e involucrantede la propia opinión.

En cuanto a la flexibilidad, se pudo observar que en la tarea "Toma de posición" la regulación con respecto a la escucha, evaluación y contra-argumentación llevó a los sujetos a relativizar sus posiciones, abandonando sus respuestas contundentes brindadas en el pre-test. Se comprende que esta relativización en los escritos implica la consideración de la existencia de puntos de vistas opuestos al propio. En la tarea "Ordenamiento" se encontró que todos los sujetos cambiaban su respuesta respecto al pre-test más allá de las condiciones testeadas, 
lo cual significaría que en este caso la flexibilidad no sería efecto de la regulación sino de la tarea en sí.

Finalmente, se pudo comprobar una cierta tendencia homogeneizante o estandarizante en las performances individuales de post-test de los sujetos de la condición con regulación en lo que hace a la amplitud argumentativa (cantidad de palabras), que aunque no llega a niveles de significatividad estadística, marca con claridad una tendencia. Este efecto homogeneizador de la amplitud argumentativa, se manifestó en los dos tipos de tarea: “Toma de posición” y "Ordenamiento". Estos resultados son similares a lo encontrado por Peralta et al. (2012) en cuanto a los efectos de la regulación sobre la homogeneización de los aprendizajes.

Como conclusión final, puede afirmarse que la variable independiente que fue objeto central de este estudio, es decir la regulación o no de la interacción diádica estimulando la escucha, la evaluación y la contra-argumentación produce efectos significativos en la competencia argumentativa individual de los sujetos, tanto en términos de complejidad como de flexibilidad y amplitud argumentativa. Sin embargo, el estudio puso de relieve también la importancia de la variable interviniente tipo de tarea ("Toma de posición” y "Ordenamiento"), la cual matiza el efecto señalado de la regulación, produciendo algunas diferenciaciones al interior de los dos tipos de tareas considerados. La importancia que tiene la familiaridad con la tarea para la elaboración de argumentos por parte de los sujetos ya ha sido destacada por Greco et al. (2017) quienes sostienen que la argumentación no puede ser escindida del tipo de tarea y contenido que se esté trabajando, sobretodo cuando están tareas son llevadas al plano del aula.

Estos resultados son importantes para el ámbito educativo, fundamentalmente en el nivel universitario, donde es posible pensar acciones concretas que permitan poner en práctica la enseñanza de la argumentación como habilidad para la formación de los estudiantes, poniendo énfasis en la estimulación de la escucha, la evaluación y la contra-argumentación. 


\section{Referencias}

Baker, M. (1996). Argumentation and co-construction des connaissainces. Interactions et cognitions, 1(2-3), 157-191.

Baker, M. (1998). Interacciones argumentativas y aprendizaje cooperativo. Escritos. Revista del Centro de Ciencias del Lenguaje, 17-18, 133-167.

Bañales Faz, G., Vega López, N, Reyna Valladares, A., Pérez Amaro, E. \& Rodríguez, B. (2014). La argumentación escrita en las disciplinas: retos de la alfabetización de los estudiantes universitarios. Revista Internacional de Ciencias Sociales y Humanidades, XXIV(2), 29-52.

Doise, W. \& Mugny, G. (1991). Percepción intelectual de un proceso histórico. Veinte años de Psicología en Ginebra: Psicología social experimental. Revista de Documentación Cientifica de la Cultura, 124, 2-23.

Felton, M., García-Milá, M., Villarroel, C. \& Gilabert, S. (2015). Arguing collaboratively: Argumentative discourse types and their potential for knowledge building. British Journal of Educational Psychology, 85(3), 372-386. https://doi.org/10.1111/bjep.12078

Felton, M., \& Kuhn, D. (2001). The development of argumentive discourse skill. Discourse Processes, 32(2-3), 135-153.

García-Milá, M., Gilbert, S., Erduran, S. \& Felton, M. (2013). The effect of argumentative task goal on the quality of argumentative discourse. Science Education, 97(4), 497- 523. https://doi.org/10.1002/sce.21057

Greco, S., Mehmeti, T. \& Perret-Clermont, A-N. (2017). Do adultchildren dialogical interactions leave space for a full development of argumentation? Journal of Argumentation in Context, 6(2), 193-219. https://doi.org/10.1075/jaic.6.2.04gre

Leitao, S. (2000). The potential of argument in knowledge building. Human Development, 43(6), 332-260. https://doi. org/10.1159/000022695 
Malpique, A., \& Viega-Simao, A. M. (2016). Escritura argumentativa en alumnos de secundaria: conocimiento sobre el discurso y rendimiento en escritura. Infancia y Aprendizaje, 39(1), 150-186. https://doi.org/10.1080/02103702.2015.1111609

Muller Mirza, N.\& Perret-Clermont, A-N.(2009). Argumentation and education. Theoretical Foundations and Practices. New York: Springer. https://doi.org/10.1007/978-0-387-98125-3

Niño, S., Castellanos, J. \& Viloria, E. (2019). Construcción del conocimiento y regulación del aprendizaje en tareas colaborativas asíncronas. Apertura, 11(1), 6-23. https://doi.org/10.32870/ Ap.v11n1.1465

Peralta, N. (2012). Investigar la interacción sociocognitiva en el ámbito educativo: recorrido teórico y resultados empíricos de un estudio en el nivel universitario. Ensemble, 4, 1-14.

Peralta, N. \& Roselli, N. (2017). Modalidad argumentativa en función del tipo de tarea y tamaño del grupo.COGENCY. Journal of Reasoning and Argumentation, 9(2), 67-83.

Peralta, N. \& Roselli, N.(2018). Estudo quase-experimental sobre argumentação em situaçóes de dilema em diferentes condiçóes de interação. Revista Eletrônica de Estudos Integrados em Discurso e Argumentação, 15, 196-208. https://doi.org/10.17648/ eidea-15-1972

Peralta, N., Roselli, N. \& Borgobello, A. (2012). El conflicto sociocognitivo como instrumento de aprendizaje en contextos colaborativos. Interdisciplinaria, 29(1), 325-338. https://doi. org/10.16888/interd.2012.29.2.8

Peralta, N. \& Castellaro, M. (2018). Interacción e intersubjetividad: investigando sus beneficios en el ámbito educativo. Caleidoscopio. Prácticas y clínicas Psi en la Universidad. Rosario: UNR editora.

Pérez-Echeverría, M., Postigo, Y. \& García-Milá, M. (2016). Argumentación y educación: apuntes para un debate. Infancia $y$ Aprendizaje, 39(1), 1-24. https://doi.org/10.1080/02103702.2 015.1111607 
Pohl, S. \& Dejean, K. (2009). Analyse de l'effect du type de tâche sur l'évolution des connaissances à la suite d'un processus d'apprentissage collaboratif. Revue internacionales de pedagogía de l'enseignement supérieur, 25(1), 1-10. https://doi.org/10.4000/ ripes. 88

Roselli, N. (1999). La construcción sociocognitiva entre iguales. Rosario: IRICE

Roselli, N. (2004). Resolución cooperativa de problemas en forma presencial y a distancia tipo chat en díadas y tríadas. Interdisciplinaria, 21(1), 71-97.

Roselli, N. (2011). Proceso de construcción colaborativa a través del chat según el tipo de tarea. Revista de Psicología, 29(1), 3-36. https://doi.org/10.18800/psico.201101.001

Recibido: 11/04/2019

Revisado: 24/07/2020

Aceptado: 27/07/2020 